\title{
Pyromellitic-Based Low Molecular Weight Gelators and Computational Studies of Intermolecular Interactions: A Potential Additive for Lubricant
}

Wannian Zhang, ${ }^{\mathrm{a}, \mathrm{b}}$, Zhiqiang Zhang, Shanlin Zhao ${ }^{\mathrm{a}, \mathrm{b}}$, Kwon Ho Hong ${ }^{\mathrm{c}}$, Ming-Yuan Zhang $^{\mathrm{b}}$, Lijuan Song ${ }^{\mathrm{a}, \mathrm{b}}$, Fang Yu ${ }^{\mathrm{b}}$, Genxiang Luo ${ }^{\mathrm{b}}$, Yu-Peng He $\mathrm{He}^{\mathrm{a}, \mathrm{b}, *}$

${ }^{a}$ Key Laboratory for Functional Material, Educational Department of Liaoning Province, School of Chemical Engineering, University of Science and Technology Liaoning, Anshan 114051, P. R. China

${ }^{\mathrm{b}}$ Key Laboratory of Petrochemical Catalytic Science and Technology, Liaoning Shihua University, Dandong Lu West 1, Fushun 113001, Liaoning, P. R. China

${ }^{\mathrm{c} D e p a r t m e n t ~ o f ~ M e d i c i n a l ~ C h e m i s t r y ~ a n d ~ I n s t i t u t e ~ f o r ~ T h e r a p e u t i c s ~ D i s c o v e r y ~ a n d ~ D e v e l o p m e n t, ~ C o l l e g e ~}$ of Pharmacy, University of Minnesota, Minneapolis 55414, United States

Corresponding author

E-mail address: yupeng.he@lnpu.edu.cn (Y.-P. He)

Table of contents

1. Synthesis of BHTA and Minimal gelator concentration----------------S2

2. Rheological properties and differential scanning calorimetry------------------S3

3. Molecular dynamics simulation and wavefunction analysis---------------S4

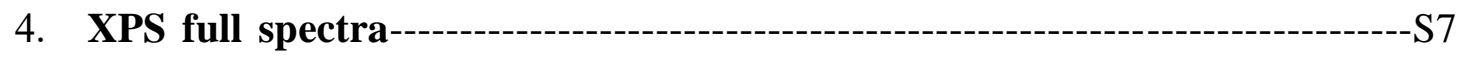




\section{Synthesis of BHTA}

2,5-bis(hexadecylcarbamoyl)terephthalic acid (BHTA) was prepared by pyromellitic dianhydride and 1-hexadecylamine. 1-hexadecylamine (4.83 g, $20.0 \mathrm{mmol})$ was added to containing pyromellitic dianhydride $(2.18 \mathrm{~g}, 10.0 \mathrm{mmol})$ acetone solution $(100 \mathrm{~mL})$. The resulting mixture was stirred at room temperature for 24 hours and filtered. The solid precipitate was filtered, Then, it was stirred in $300 \mathrm{ml}$ ethanol for 1 hours and filtered, this process was repeated for three times. Then, the product was next stirred in $300 \mathrm{ml}$ acetone for 1 hours and filtered, this process was also repeated for three times. The clean product was dried to give a white powder in $36 \%$ yield. Melting point: $196^{\circ} \mathrm{C}$; NMR (DMSO-d 6,400 MHz): $\delta=13.23$ (br. 2H), 8.45 (s, 1H), $7.68(\mathrm{~s}, 1 \mathrm{H}), 3.18$ (br, $4 \mathrm{H}), 1.49$ (br, 4H), 1.24 (br, 52H), 0.85 (br, 6H); HR-MS Calcd for $\mathrm{C}_{42} \mathrm{H}_{73} \mathrm{~N}_{2} \mathrm{O}_{6}{ }^{+}$ $[\mathrm{M}+\mathrm{H}]^{+}:$701.5463; Found: 701.5467.

Melting Point was operated on Beijing Taike Instrument, X-6. NMR spectra was recorded on Bruker Ultrashield ${ }^{\mathrm{TM}} 400$ Plus at $60^{\circ} \mathrm{C}$ and calibrated using DMSO peaks as internal reference. Multiplicities are recorded as: $\mathrm{s}=$ singlet, $\mathrm{br}=$ broad peak. High resolution ESI mass experiments were operated on a Bruker Daltonics, Inc. APEXIII 7.0 TESLA FTMS instrument.

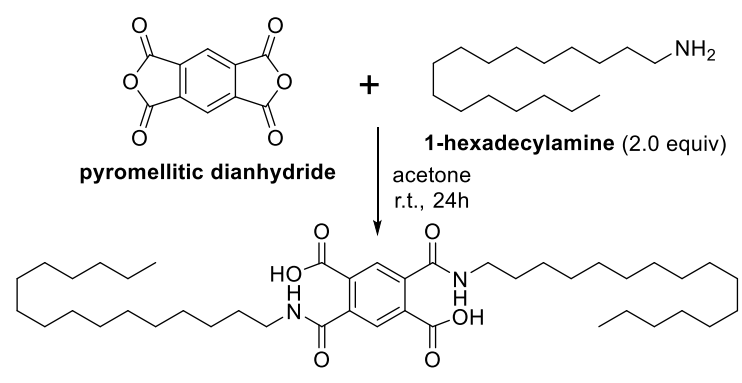

2,5-bis(hexadecylcarbamoyl)terephthalic acid (BHTA)

Scheme S1: Synthesis route of gelator BHTA

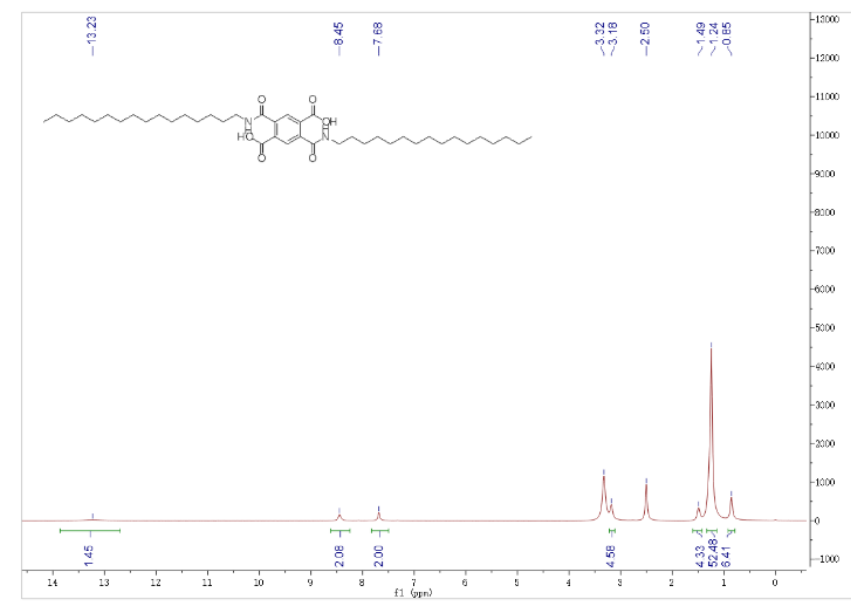

Figure S1: 1H-NMR of BHTA 
Table S1. Minimal gelator concentration (MGC, wt\%) of organogelators BHTA in solvent

\begin{tabular}{lccccccc}
\hline & $n$-hexadecane & diese $^{a}$ & liquid paraffin & $150 \mathrm{BS}$ & $500 \mathrm{SN}$ & PAO10 & PAO40 \\
\hline MGC & 0.47 & 0.84 & 0.51 & 1.12 & 1.54 & 2.09 & 2.41 \\
\hline
\end{tabular}

${ }^{a}$ The diesel oil is $35^{\#}$ diesel (China standard diesel GB/T19147-2013).

\section{Rheological properties and differential scanning calorimetry}

Oscillatory stress and angular frequency mode experiments were carried out to study the rheological behaviors of these organogels and investigate the mechanical strength for potential applications (Figure S2). The elastic modulus (color red, G') of the gels from $n$-hexadecane decreased rapidly and fell below the viscous modulus (color blue, G', ) after the critical strain region, indicating the gels underwent a gel-sol transformation. The frequency sweeps shown that the elastic modulus G' was greater than the viscous modulus G', over the frequency range $(0.01-100 \mathrm{rad} / \mathrm{s})$ for the gels $(\gamma$ fixed at $0.05 \%$ ). This observation confirmed the formation of soft solid-like gels. Similar results have been showed for the gels of liquid paraffin, diesel oil and 150BS.

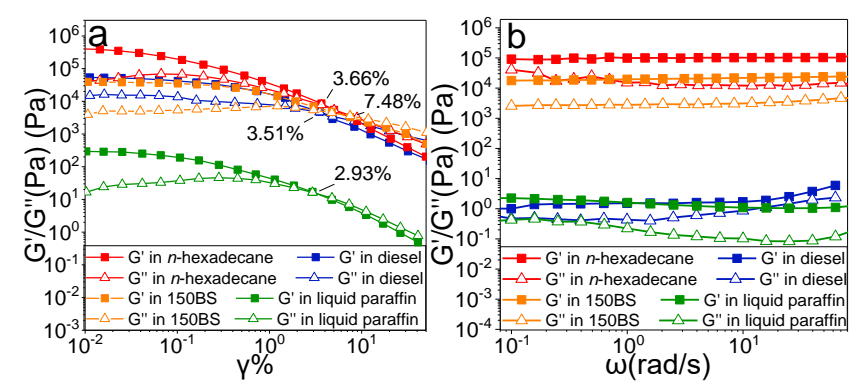

Figure S2. Rheological studies for the diesel gels of BHTA (3 times MGC): (a) stress sweep, (b) frequency sweep.

Thermal stability of gels formed by BHTA in diesel oil, liquid paraffin, $n$-hexadecane and 150BS have been tested based on their gel-to-sol transition temperatures, Tgel from the differential scanning calorimetry (DSC) study (Figure S3). In diesel, Tgel values were $43.2{ }^{\circ} \mathrm{C}$ and $67.9{ }^{\circ} \mathrm{C}$ for 3 folds MGC amount of BHTA. In paraffin oil, Tgel values were $66.4{ }^{\circ} \mathrm{C}$ and $85.1{ }^{\circ} \mathrm{C}$ for 3 folds MGC amount of BHTA. In $n$-hexadecane, Tgel values were $80.5{ }^{\circ} \mathrm{C}$ and $92.9{ }^{\circ} \mathrm{C}$ for 3 folds MGC amount of BHTA. In 150BS, Tgel values were $62.4{ }^{\circ} \mathrm{C}$ and $95.3{ }^{\circ} \mathrm{C}$ for 3 folds MGC amount of BHTA. These data showed that BHTA has a high thermodynamic stability in diesel oil, liquid paraffin, $n$-hexadecane and 150BS. 

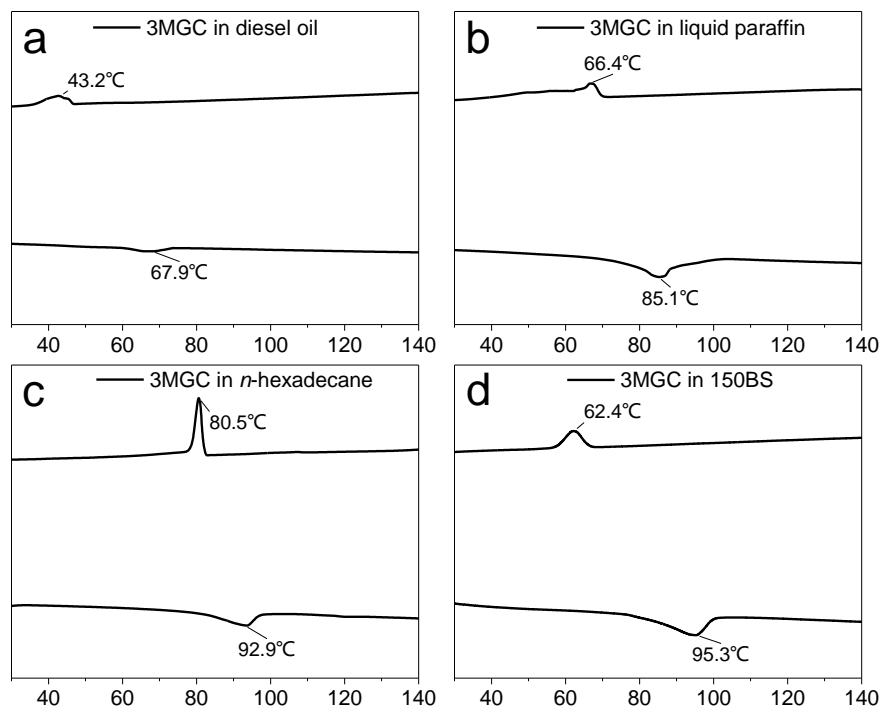

Figure S3. DSC studies for gels of BHTA (3 folds of MGC). (a) in diesel oil; (b) in liquid paraffin, (c) in $n$-hexadecane; (d) in 150BS gels.

\section{Molecular dynamics simulation and wavefunction analysis}

In order to evaluate the main driving force of self-assembly, MD simulations were performed with BHTA molecules. The single BHTA structure was subject to Gaussian 16 Rev. A.03 program at M06-2X/6-31+G(d,p) level for geometry optimization. First, the d-spacing between single BHTA molecule was detected to be $0.45 \mathrm{~nm}$ according to XRD value. Then, columnar aggregates containing 20 BHTA molecules (approximate to 3 times MGC) in periodic boxes of $8 * 8 * 15 \mathrm{~nm}^{3}$ were built by PACKMOL program, then filled with $1800 n$-hexadecane solvent molecules to keep the natural density. After that, it is directly used as the starting point to start the simulation calculation. 


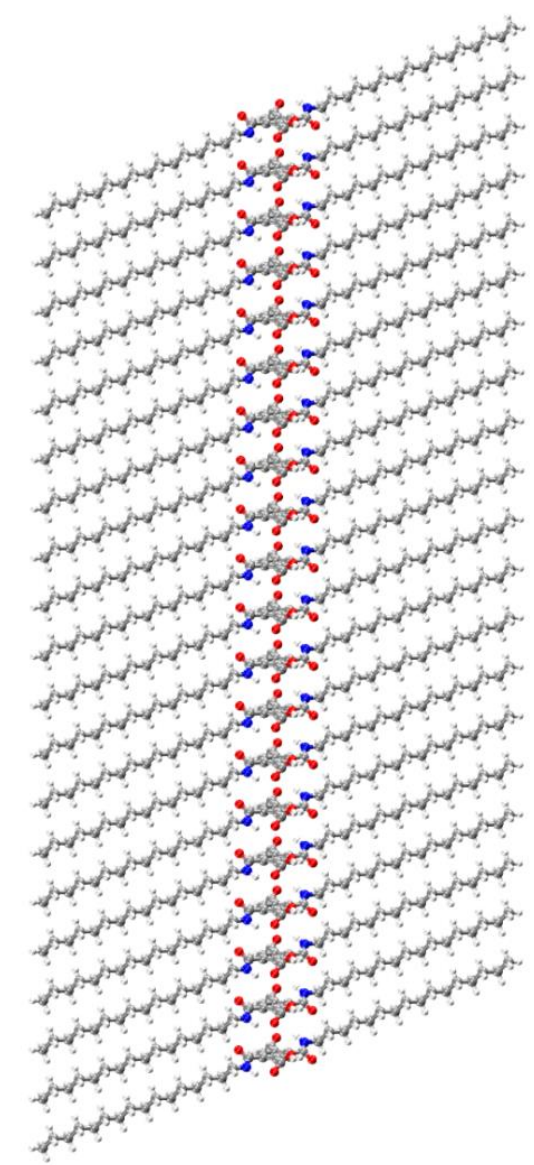

Figure S4: Initial MD simulation model

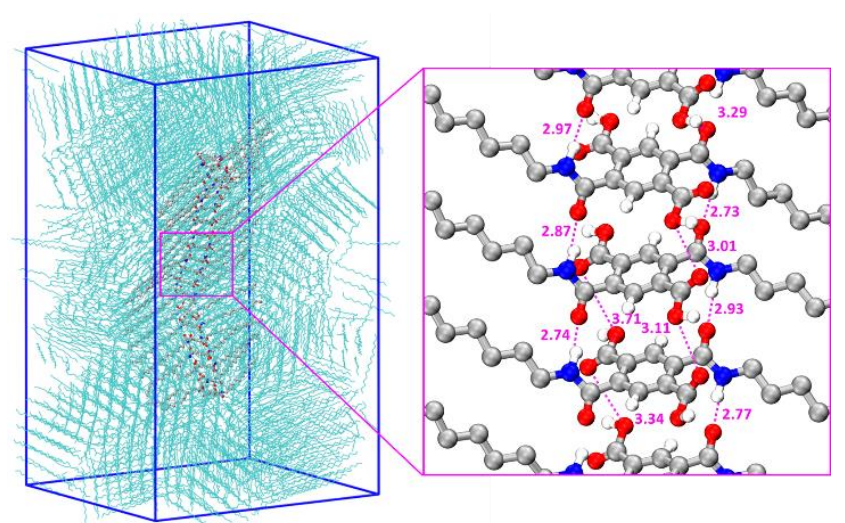

Figure S5. Original MD simulation model of BHTA in $n$-hexadecane in periodic boxes of $8 * 8 * 15$ $\mathrm{nm}^{3}$. Unit of hydrogen bond distance: $\AA$. 


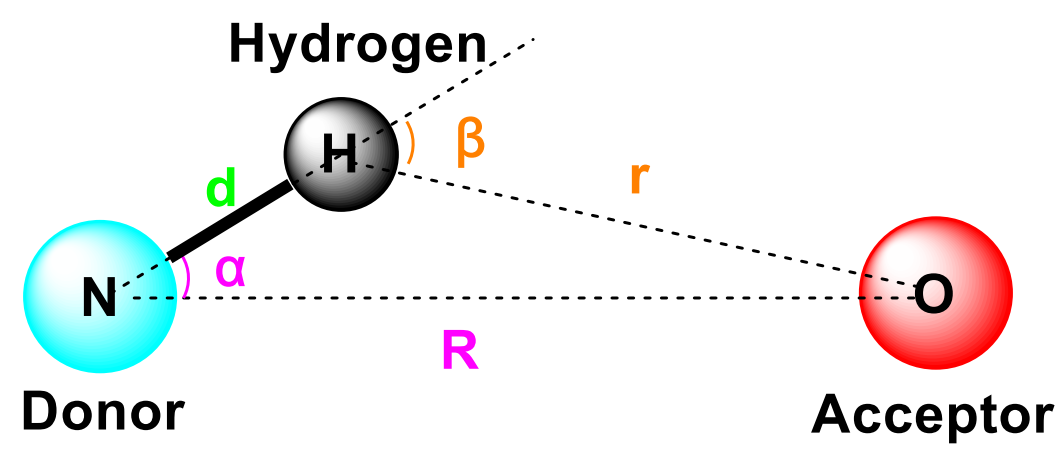

Figure S6: H-bond is GROMACS' command to count the H-bonds in the trajectory according to the geometric standard. O-H and $\mathrm{N}-\mathrm{H}$ are used as $\mathrm{H}$-bond donors, and $\mathrm{O}$ and $\mathrm{N}$ are used as H-bond acceptors. The statistics on the distance distribution of H-bonds ( $R$ ) in this article are the distances between heavy atoms and heavy atoms.

Energy decomposition also shed light to mechanistic study of self-assembly from the energy point of view. The Figure S7 shows the energy decomposition of the BHTA aggregates after the MD simulation of the original model. The energy data were attracted from 15-30 ns during the equilibrium state. The energy contribution was $-13431 \mathrm{~kJ} / \mathrm{mol}$ for electrostatic action vs -2264 $\mathrm{kJ} / \mathrm{mol}$ for $v \mathrm{dW}$ effect. This data indicated the main form of action between the BHTA is electrostatic action and contributes to the intense hydrogen bonding network required for selfassembly of the gel aggregates. The form of the effect between the BHTA and $n$-hexadecane is mainly dispersion effect (-6621 vs $46 \mathrm{~kJ} / \mathrm{mol}$ for electrostatic action), and the dispersion attraction in solvent molecules ( $n$-hexadecane) is overwhelmingly strong $(-178313 \mathrm{~kJ} / \mathrm{mol})$, while the electrostatic effect $(23734 \mathrm{~kJ} / \mathrm{mol})$ as a penelty for self-assembly between solvent molecules.

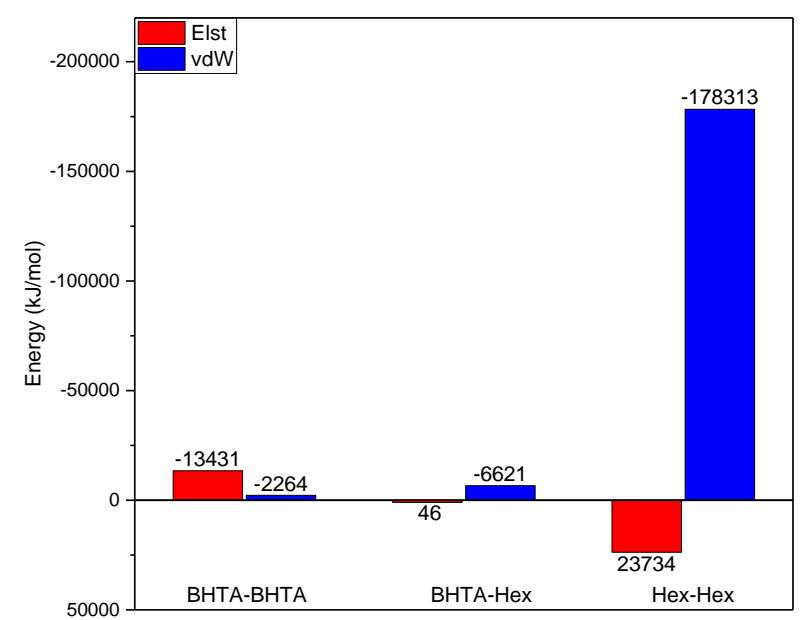

Figure S7. Energy decomposition diagram. Hex stands for $n$-hexadecane: Elst (electrostatic, red) and vdW (van der Waals, blue). 
4. XPS full spectra
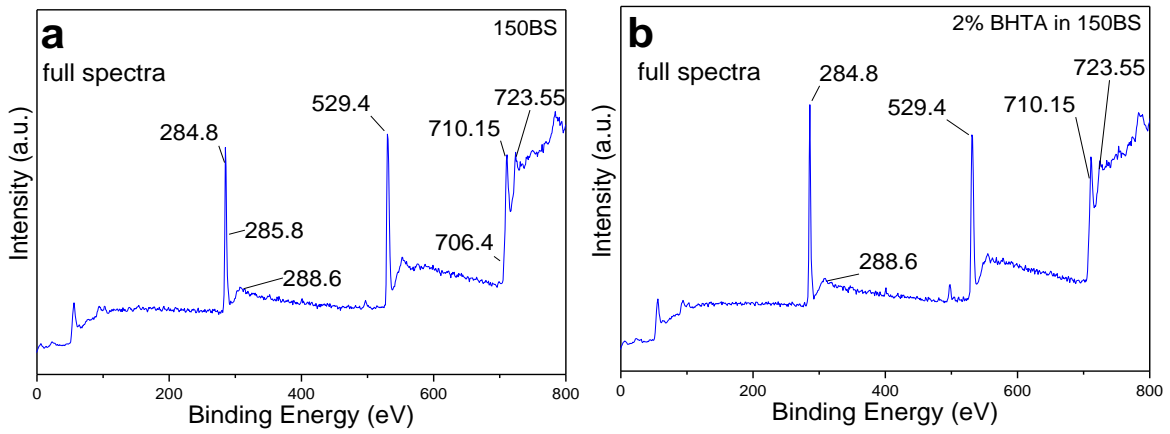

Figure S8. XPS full spectra of the worn surfaces lubricated: (a) by 150BS; (b) by gel lubricant with $2 \mathrm{wt} \%$ BHTA in 150BS. 